\title{
On the Distribution of Indefinite Quadratic Forms in Gaussian Random Variables
}

\author{
Tareq Y. Al-Naffouri \\ Department of Electrical Engineering \\ King Fahd University of Petroleum \& Minerals \\ Dhahran, Saudi Arabia \\ Email: naffouri@kfupm.edu.sa
}

\author{
Babak Hassibi \\ Department of Electrical Engineering \\ California Institute of Technology \\ Pasadena, CA \\ Email: hassibi@systems.caltech.edu
}

\begin{abstract}
In this work, we propose a transparent approach to evaluating the CDF of indefinite quadratic forms in Gaussian random variables and ratios of such forms. This quantity appears in the analysis of different receivers in communication systems and in various applications in signal processing. Instead of attempting to find the pdf of this quantity as is the case in many papers in literature, we focus on finding the CDF. The basic trick that we implement is to replace inequalities that appear in the CDF calculations with the unit step function and replace the latter with its Fourier transform. This produces a multi-dimensional integral that can be evaluated using complex integration. We show how our approach extends to nonzero mean Gaussian real/complex vectors and to the joint distribution of indefinite quadratic forms. ${ }^{1}$
\end{abstract}

\section{INTRODUCTION AND PROBLEM DEFINITION}

Gaussian random variables play a very important role in signal processing, communications, and information theory. It is very important to find the distributions of various quantities involving Gaussian random variables, most notably sums of squares of Gaussian random variables and ratios of such norms. Such quantities appear in many applications including for example mean-square-error (MSE) analysis of the normalized least-mean-square (NLMS) algorithm, SINR calculations, and maximum ratio combining (MRC) diversity schemes in CDMA systems.

A considerable amount of research has been done to find the distribution of quadratic forms in Gaussian random variables. However, these approaches are either restricted to special cases (for example, positive definite case [1], [2], central case [3], real Gaussian random variables case [4], [5], [6], [7], ... etc.), or provide highly complex solutions using series expansion or approximations for indefinite quadratic forms [3], [8], [4], [6], [9], [10], [11], [12], [13], [2], [14], [15], [16], [17]. Numerical integration is used in [18], [19] to evaluate the distribution of an indefinite quadratic forms. Because of these highly complex solutions and the treatment of special cases, their practical usefulness is limited. A third disadvantage of these methods is their focus on obtaining the pdf from the characteristic function when the CDF is a more useful expression. The reason is that the CDF (just like the pdf) can be used to

\footnotetext{
${ }^{1}$ The work of T. Y. Al-Naffouri was supported by the Fulbright Scholar Program and by a research grant from King Fahd University of Petroleum and Minerals, Dhahran, Saudi Arabia.
}

obtain the moments (through integration by parts). Moreover, the CDF directly gives an expression for the probability (when the pdf needs to be integrated to obtain this information).

The aim of this paper is to introduce a new and effective method for characterizing the statistical behavior of indefinite quadratic forms in Gaussian r.v.'s (i.e., to obtain an expression for the cumulative distribution function (CDF) of indefinite quadratic forms).

In this article, we study the distribution of various quantities involving weighted norms of a correlated Gaussian vector and show how to find the distribution of these quantities using complex integration. Calculating the CDF involves integrating the $M$-dimensional Gaussian over a region defined by an inequality constraint. Our approach simply relies on replacing this constraint with the unit step function thus transforming the constrained integral into an unconstrained one. To deal with the unit step function, we replace it with its Fourier Transform creating an $M+1$ dimensional integral with infinite limits. The advantage of this approach is that all manipulations can be done within the integrand itself. Complex integration can now be used to calculate the integral. Other advantages of our approach are detailed below.

1) The approach relies on directly finding the CDF as opposed to finding the pdf through the usual characteristic function approach (which requires two integration steps).

2) The approach applies to general indefinite quadratic forms of correlated Gaussian random vectors (complex or real).

3) We obtain the CDF of the quadratic form as a finite sum of expenentials (as opposed to the infinite summation expression obtained in [17]). The exponent is the eignevalue of the matrix of the resulting indefinite form.

4) Our approach applies to non-zero mean random variables although the result can be evaluated as a onedimensional integral.

5) The same approach can be used to evaluate the joint distribution of indefinite quadratic forms.

6) Our approach applies to other variables including for example indefinite quadratic forms in isotropically distributed variables. 
7) The approach is not limited to indefinite quadratic forms but can be extended to other (non)quadratic forms of (non)Gaussian distributions.

\section{Problem Formulation}

Let $A$ be a Hermitian matrix of size $M$ and consider the random quadratic form

$$
\begin{aligned}
Y & =\|H\|_{A}^{2} \\
& \triangleq H^{*} A H
\end{aligned}
$$

where $H \sim C \mathcal{N}(0, R)$. Without loss of generality, we have assumed that $H$ is white. To see this, define $H_{w}=R^{\frac{1}{2}} H$, then $H_{w}$ is white and

$$
\|H\|_{A}^{2}=\left\|H_{w}\right\|_{R^{\frac{1}{2}} A R^{\frac{*}{2}}}^{2}
$$

When $H$ is non-zero mean we can equivalently consider the (noncentral) quadratic form

$$
Y_{n c}=\|H-m\|_{A}^{2}
$$

For real quadratic forms, we have

$$
Y_{r}=\left\|H_{r}\right\|_{A_{r}}^{2} \triangleq H_{r}^{\prime} A_{r} H_{r}
$$

where $H_{r}$ is a white real Gaussian vector and $A_{r}$ is a symmetric real matrix.

\section{The Distribution of an Indefinite Hermitian QUADRATIC FORM}

Consider the random Hermitian quadratic form $Y=\|H\|_{A}^{2}$. The CDF of $Y$ is given by

$$
\begin{aligned}
F_{Y}(y) & =P\{Y \leq y\} \\
& =\int_{\mathcal{A}} p\left(H_{i}\right) d H_{i}
\end{aligned}
$$

where $\mathcal{A}$ is the area in $M$ multidimensional $H$ plane defined by the inequality $\|H\|_{A}^{2} \leq y$. The integral as it appears above is difficult to evaluate. An alternative way to do so is to express the inequality that appears in (6) as

$$
y-\|H\|_{A}^{2} \geq 0
$$

So, the CDF takes the form

$$
F_{Y}(y)=\frac{1}{2 \pi^{M}} \int_{-\infty}^{\infty} e^{-H^{*} H} u\left(y-\|H\|_{A}^{2}\right) d H
$$

In the integral above, $u$ is the unit step function and the integration is performed over the entire $H$ plane, transforming all operations into the integrand. The above integral is still difficult to evaluate. So, we replace the unit step by its Fourier transform

$$
u(x)=\frac{1}{2 \pi} \int_{-\infty}^{\infty} \frac{e^{x(j \omega+\beta)}}{j \omega+\beta} d \omega
$$

which is valid for any $\beta>0$ (and is also independent of the value of $\beta)^{2}$. This yields the the following $M+1$ dimensional integral

$F_{Y}(y)=\frac{1}{2 \pi^{M+1}} \int d \omega \frac{e^{y(j \omega+\beta)}}{j \omega+\beta} \int d H e^{-H^{*}(I+A(j \omega+\beta)) H}$

By examining this integral, we note that the inner integral looks like a Gaussian integral. Intuition suggests that this integral can be written as (see the Appendix for a formal proof)

$$
\frac{1}{\pi^{M}} \int e^{-H^{*}(I+A(j \omega+\beta)) H} d H=\frac{1}{\operatorname{det}(I+A(j \omega+\beta))}
$$

provided $\beta$ is chosen small enough to make $I+\beta A$ positive definite. Thus, we can express the $\operatorname{CDF}$ of $Y$ as

$$
F_{Y}(y)=\frac{1}{2 \pi} \int_{-\infty}^{\infty} \frac{e^{y(j \omega+\beta)}}{j \omega+\beta} \prod_{i=1}^{M} \frac{1}{1+\lambda_{i}(j \omega+\beta)} d \omega
$$

where the $\lambda_{i}^{\prime} \mathrm{s}$ are the eigenvalues of $A$. This reduces the $M+1$ dimensional integral into a one-dimensional integration problem in the variable $j \omega+\beta$. To evaluate this integral, we need to first expand the fraction that appears above in a partial fraction expansion. Thus, assume that $A$ has exactly $L$ distinct eigenvalues $\lambda_{1}, \ldots, \lambda_{L}$ where $\lambda_{l}$ has multiplicity $K_{l}$. Then the fraction in (10) can be expanded as (for some constants $\alpha_{k_{l}}$ )

$$
\begin{array}{r}
\frac{1}{j \omega+\beta} \frac{1}{\prod_{i=1}^{M}\left(1+\lambda_{i}(j \omega+\beta)\right)}=\frac{1}{j \omega+\beta}+ \\
\sum_{l=1}^{L} \sum_{k_{l}=0}^{K_{l}} \frac{\alpha_{k_{l}}}{1+\lambda_{i}(j \omega+\beta)}
\end{array}
$$

Now, using residue value theory, we can show that [20]

$$
\begin{aligned}
\frac{1}{2 \pi} \int_{-\infty}^{\infty} \frac{e^{+j \omega p}}{(a+j \omega)^{\nu}} d \omega & =\left\{\begin{array}{cc}
\frac{p^{\nu-1}}{\Gamma(\nu)} e^{-a p} u(p) & a>0 \\
-\frac{(-p)^{\nu-1}}{\Gamma(\nu)} e^{-a p} u(-p) & a<0
\end{array}\right. \\
& =\frac{\operatorname{sign}^{\nu-1}(a)}{\Gamma(\nu)}(p)^{\nu-1} e^{-a p} u(a p)
\end{aligned}
$$

We can use this to evaluate the $1+L\left(K_{l}+1\right)$ integrals of (10)-(11). Specifically, we have

$$
\frac{1}{2 \pi} \int_{-\infty}^{\infty} \frac{e^{x(j \omega+\beta)}}{j \omega+\beta} d \omega=e^{\beta x} e^{-\beta x} u(x)=u(x)
$$

and

$$
\begin{aligned}
\frac{1}{2 \pi} \int_{-\infty}^{\infty} \frac{e^{y(j \omega+\beta)}}{1+\lambda_{i}(j \omega+\beta)} & =\frac{e^{y \beta}}{\lambda_{i}^{k_{l}}} \frac{1}{2 \pi} \int_{-\infty}^{\infty} \frac{e^{y j \omega}}{\left(\beta+\frac{1}{\lambda_{i}}+j \omega\right)^{k_{l}}} \\
& =\frac{1}{\Gamma\left(k_{l}\right)\left|\lambda_{l}\right|^{k_{l}}} y^{k_{l}-1} e^{-\frac{y}{\lambda_{l}}} u\left(\frac{y}{\lambda_{l}}\right)
\end{aligned}
$$

where in arriving at the last integral, we used the fact that $\beta$ was chosen so that $1+\beta \lambda_{i}>0$, i.e., $\operatorname{sign}\left(\beta+\frac{1}{\lambda_{i}}\right)=\operatorname{sign}\left(\lambda_{i}\right)$. Note that both integrals in (12) and (13) are independent of $\beta$

\footnotetext{
${ }^{2}$ The value of $\beta$ is chosen judicially to help evaluate certain multidimensional integrals of the Gaussian pdf.
} 
as they should. This allows us to write the $\operatorname{CDF} F_{Y}(y)$ in the following closed form

$$
F_{Y}(y)=u(y)+\sum_{l=1}^{L} \sum_{k_{l}=1}^{K_{l}} \frac{\alpha_{k_{l}}}{\Gamma\left(k_{l}\right)\left|\lambda_{l}\right|^{k_{l}}} y^{k_{l}-1} e^{-\frac{y}{\lambda_{l}}} u\left(\frac{y}{\lambda_{l}}\right)
$$

When none of the eigenvalues is repeated, the CDF takes the form

$$
F_{Y}(y)=u(y)-\sum_{i=1}^{M} \frac{\lambda_{i}^{M}}{\prod_{l \neq i}\left(\lambda_{i}-\lambda_{l}\right)} \frac{1}{\left|\lambda_{i}\right|} e^{-\frac{y}{\lambda_{i}}} u\left(\frac{y}{\lambda_{i}}\right)
$$

\section{A. The distribution of a ratio of Gaussian norms}

Let's apply the result developed above to derive the CDF of the ratio

$$
X=\frac{\epsilon_{1}+\|H\|_{B_{1}}^{2}}{\epsilon_{2}+\|H\|_{B_{2}}^{2}}
$$

where $B_{1}$ and $B_{2}$ are Hermitian. Such a quantity appears in numerous applications in signal processing and communications (e.g., in the analysis of normalized adaptive filters [21] and in SINR calculations [22], [23]). Now note that the probability

$$
P\left\{\frac{\epsilon_{1}+\|H\|_{B_{1}}^{2}}{\epsilon_{2}+\|H\|_{B_{2}}^{2}} \leq x\right\}
$$

can be equivalently written as

$$
P\left\{\|H\|_{B_{1}-x B_{2}} \leq \epsilon_{2} x-\epsilon_{1}\right\}
$$

which is of the same form described above for

$$
A=B_{1}-x B_{2} \text { and } y=\epsilon_{2} x-\epsilon_{1}
$$

and so we can immediately deduce the CDF to be

$$
\begin{aligned}
F_{Y}\left(\epsilon_{2} x-\epsilon_{1}\right)=u\left(\epsilon_{2} x-\epsilon_{1}\right)-\sum_{i=1}^{M} \frac{\lambda_{i}^{M}(x)}{\prod_{l \neq i}\left(\lambda_{i}(x)-\lambda_{l}(x)\right)} \\
\times \frac{1}{\left|\lambda_{i}(x)\right|} e^{-\frac{\epsilon_{2} x-\epsilon_{1}}{\lambda_{i}}} u\left(\frac{\epsilon_{2} x-\epsilon_{1}}{\lambda_{i}}\right)
\end{aligned}
$$

Here $\lambda_{i}(x) i=1, \ldots, M$ are the eigenvalues of $B_{1}-x B_{2}$ and hence are functions of $x$. The expression (16) is valid assuming that the eigenvalues of $B_{1}-x B_{2}$ are distinct for each $x$.

To get an intuition of when this is true, consider the special case when both $B_{1}$ and $B_{2}$ are diagonal. In this case

$$
\lambda_{i}(x)=b_{1_{i}}-b_{2_{i}} x
$$

and $\lambda_{i}(x)=\lambda_{j}(x)$ provided that

$$
b_{1_{i}}-b_{1_{j}}=\left(b_{2_{i}}-b_{2_{j}}\right) y
$$

If $b_{2_{i}} \neq b_{2_{j}}$, this happens at $x=\frac{b_{1_{i}}-b_{1_{j}}}{b_{2_{i}}-b_{2_{j}}}$, an event that occurs with zero probability. Alternatively, if $b_{1_{i}}=b_{1_{j}}$ and $b_{2_{i}}=b_{2_{j}}$, then $\lambda_{i}(x)=\lambda_{j}(x)$ for all $x$. For general matrices $B_{1}$ and $B_{2}$, the condition $\lambda_{i}(x)=\lambda_{j}(x)$ is satisfied for all $x$ provided that both $B_{1}$ and $B_{2}$ have repeated eigenvalues and share the same corresponding eigenvector (see [24] for a proof).

Figure 1 shows the empirical and analytical CDF $X$ defined in (14) where $H$ is white Gaussian of size $10 \times 1$ and $B_{1}$ and $B_{2}$ are randomly chosen fixed matrices of size 10 . In the simulations, we set $\epsilon_{1}=\epsilon_{2}=0.01$. The figure shows excellent match between the analytical expression and the simulated CDF.

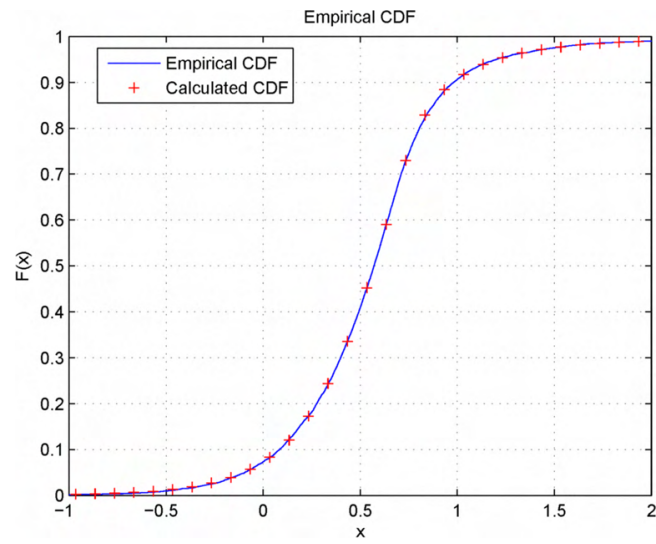

Fig. 1. Empirical CDF vs Calculated PDF of $X$

\section{B. The Nonzero-Mean Case}

In the above, we assumed the Gaussian variables to be of zero mean. Let's consider Hermitian quadratic forms in nonzero mean Gaussian vectors. Equivalently, we would like to consider the non-central quadratic form

$$
Y=\|H-a\|_{A}^{2}
$$

where $H \sim \mathcal{N}(0, I)$. Let's find the probability $\operatorname{Pr}\{Y \leq y\}$. Along the lines pursued above, we can write this probability as the $M+1$ dimensional integral

$$
\begin{aligned}
\operatorname{Pr}\{Y \leq y\}= & \frac{1}{\pi^{M}} \int_{-\infty}^{\infty} e^{-\|H\|^{2} u\left(y-\|H-a\|_{A}^{2}\right)} d H \\
= & \frac{1}{2 \pi^{M+1}} \int_{-\infty}^{\infty} d \omega \frac{e^{y(j \omega+\beta)}}{j \omega+\beta} \times \\
& \int d H e^{-\left(\|H\|^{2}+\|H\|_{(j \omega+\beta) A}^{2}\right)} d H(17)
\end{aligned}
$$

By completing the squares, we can write the sum of (weighted) norms that appears above as a single (noncentral) quadratic form

$$
\|H\|^{2}+\|H-a\|_{(j \omega+\beta) A}^{2}=\|H-b\|_{B}^{2}+c
$$

where

$$
\begin{aligned}
b & =\left(I+\frac{1}{j \omega+\beta} A^{-1}\right)^{-1} a \\
B & =I+(j \omega+\beta) A \\
c & =a^{*}\left(I+\frac{1}{j \omega+\beta} A^{-1}\right)^{-1} a
\end{aligned}
$$

which allows us to reduce (17) into a 1-dimensional integral

$\operatorname{Pr}\{Y \leq y\}=\frac{1}{2 \pi} \int_{-\infty}^{\infty} \frac{e^{y(j \omega+\beta)}}{j \omega+\beta} e^{-c} \frac{1}{\operatorname{det}(I+(j \omega+\beta) A)} d \omega$ 
Now, let $A=Q^{*} \Lambda Q$ denote the eigenvalue decomposition of $A$. Assuming that non of the eigenvalues of $A$ are repeated, we can rewrite the integral above as a sum of simpler integrals by relying on the partial fraction expansion

$$
\begin{aligned}
& \operatorname{Pr}\{Y \leq y\}=\frac{1}{2 \pi} \int_{-\infty}^{\infty} \frac{e^{y(j \omega+\beta)}}{j \omega+\beta} e^{-c} d \omega+ \\
& \sum_{i=1}^{M} \frac{1}{2 \pi \prod_{l \neq i}\left(1-\frac{\lambda_{l}}{\lambda_{i}}\right)} \int_{-\infty}^{\infty} \frac{e^{y(j \omega+\beta)}}{1+\lambda_{i}(j \omega+\beta)} e^{-c} d \omega
\end{aligned}
$$

As, we can see, we need to evaluate $M+1$ integrals of the form

$$
\begin{aligned}
I_{i} & =\frac{1}{2 \pi} \int_{-\infty}^{\infty} \frac{e^{y(j \omega+\beta)}}{1+\lambda_{i}(j \omega+\beta)} e^{-c} d \omega \\
& =\frac{1}{2 \pi} \int_{-\infty}^{\infty} \frac{e^{y(j \omega+\beta)}}{1+\lambda_{i}(j \omega+\beta)} e^{-\sum_{m=1}^{M} \frac{|\tilde{a}(m)|^{2}(j \omega+\beta) \lambda_{m}}{1+(j \omega+\beta) \lambda_{m}}} d \omega
\end{aligned}
$$

where $\tilde{a}=Q a$. It is not possible to evaluate this integral in closed form (except when all the eigenvalues are equal), but we can evaluate it numerically.

\section{The Real Quadratic Form Case}

Let's now consider the case when the quadratic form is in real Gaussian variable. In this case, we arrive at the same expressions in Section II except that conjugate transpose is replaced with transpose and the matrix $A$ is now real symmetric

$$
F_{Y}(y)=\frac{1}{2 \pi^{M+1}} \int d \omega \frac{e^{y(j \omega+\beta)}}{j \omega+\beta} \int d H e^{-H^{T}(I+A(j \omega+\beta)) H} d H
$$

The inner integral now integrates to $\frac{1}{\sqrt{(I+A(j \omega+\beta))}}$ and this leads to the 1-dimensional integral

$\operatorname{Pr}\{Y \leq y\}=\frac{1}{2 \pi} \int_{-\infty}^{\infty} \frac{e^{y(j \omega+\beta)}}{j \omega+\beta} \frac{1}{\sqrt{\operatorname{det}(I+A(j \omega+\beta))}} d \omega$

This integral can not be evaluated in closed form in general and can instead be evaluated numerically.

\section{JOINT Distributions OF INDEFINITE HERMITIAN QUADRATIC FORMS}

We can use the same method considered above to find the joint distribution of several quadratic forms. We shall demonstrate this here for the two quadratic forms case, although our insights can be easily extended to more than two quadratic forms. Thus, consider the CDF

$$
F_{X_{a}, X_{b}}\left(x_{a}, x_{b}\right)=\operatorname{Pr}\left\{\|H\|_{A}^{2} \leq x_{a},\|H\|_{B}^{2} \leq x_{b}\right\}
$$

By representing the inequalities $x_{a}-\|H\|_{A}^{2} \geq 0$ and $x_{b}-$ $\|H\|_{B}^{2} \geq 0$ as step functions and further replacing the step functions using the integral representation (8), we can write the $\mathrm{CDF}$ above as the $M+2$ dimensional integral

$$
\begin{aligned}
F_{X_{a}, X_{b}}\left(x_{a}, x_{b}\right)= & \frac{1}{2^{2} \pi^{M+2}} \int d \omega_{1} \frac{e^{x_{a}\left(j \omega_{1}+\beta_{1}\right)}}{j \omega_{1}+\beta_{1}} \\
& \int d \omega_{2} \frac{e^{x_{b}\left(j \omega_{2}+\beta_{2}\right)}}{j \omega_{2}+\beta_{2}} \int d H \\
& e^{-\|H\|^{2}-\|H\|_{\left(j \omega_{1}+\beta_{1}\right) A}^{2}-\|H\|_{\left(j \omega_{2}+\beta_{2}\right) B}^{2}}
\end{aligned}
$$

The $H$-dependent part of the integral can be represented as

$$
I_{H}=\frac{1}{\pi^{M}} \int d H e^{-\|H\|_{I+\left(j \omega_{1}+\beta_{1}\right) A+\left(j \omega_{2}+\beta_{2}\right) B}^{2}}
$$

Now, just as we argued in Appendix A, $\beta_{1}$ and $\beta_{2}$ can be chosen to make the real part of the weight above positive definite and so (18) reduces to

$$
I_{H}=\frac{1}{\operatorname{det}\left(I+\left(j \omega_{1}+\beta_{1}\right) A+\left(j \omega_{2}+\beta_{2}\right) B\right)}
$$

so that

$$
\begin{aligned}
F_{X_{a}, X_{b}}\left(x_{a}, x_{b}\right)= & \frac{1}{2^{2} \pi^{2}} \int d \omega_{1} \frac{e^{x_{a}\left(j \omega_{1}+\beta_{1}\right)}}{j \omega_{1}+\beta_{1}} \\
& \int d \omega_{2} \frac{e^{x_{b}\left(j \omega_{2}+\beta_{2}\right)}}{j \omega_{2}+\beta_{2}} \\
& \frac{1}{\operatorname{det}\left(I+\left(j \omega_{1}+\beta_{1}\right) A+\left(j \omega_{2}+\beta_{2}\right) B\right)}
\end{aligned}
$$

In general, we can not evaluate this integral in closed form unless $A$ and $B$ are jointly diagonalizable by an orthonormal transformation or equivalently that $A$ and $B$ are diagonal. Under this assupmtion, the determinant in (19) can be easily expanded and the joint CDF takes the form

$$
\begin{aligned}
F_{X_{a}, X_{b}}=\frac{1}{2^{2} \pi^{2}} \int d \omega_{1} \frac{e^{x_{a}\left(j \omega_{1}+\beta_{1}\right)}}{j \omega_{1}+\beta_{1}} \int d \omega_{2} \frac{e^{x_{b}\left(j \omega_{2}+\beta_{2}\right)}}{j \omega_{2}+\beta_{2}} & \frac{1}{\prod_{i=1}^{M}\left(I+\left(j \omega_{1}+\beta_{1}\right) a_{i}+\left(j \omega_{2}+\beta_{2}\right) b_{i}\right)}
\end{aligned}
$$

Now it is tedious but straightforward to evaluate this double integral in closed form. We consider the fraction that appears in (20) as a function in $j \omega_{2}+\beta_{2}$ and expand it in a partial fraction expansion. This results in $M+1$ terms (assuming that that non of the terms are repeated). Each of these terms can be integrated with respect to $\omega_{2}$ to produce $M+1$ terms that are in turn partial fractions in $j \omega_{1}+\beta_{1}$. The same process can now be repeated for the $\omega_{1}$ variable, arriving finally at a closed form expression for the CDF.

\section{CONCLUSION}

In this paper, we derived the distribution of indefinite quadratic forms in Gaussian random variables. As opposed to the many studies in literature that considered this problem, we avoided the traditional "characteristic function to pdf" approach and instead focused on finding the CDF directly. The CDF is a more useful expression than the pdf since it gives the probability value directly and since it can be used to obtain the moments using integration (by parts) just as is the case for the pdf. Our approach relies on representing the inequalities that naturally appear in CDF calculations using the unit step function and representing the latter using its Fourier transform. Our approach is unified in that it equally applies to the real and complex cases and to the zero and nonzero mean cases. It can be naturally extended to joint CDF calculations. While, we have not shown that in this paper, our approach can be used to obtain bounds on the CDF and also to find the CDF 
of (non) quadratic forms in (non) Gaussian random variables [24].

\section{APPENDIX}

In this appendix, we prove our in claim in (9) that

$$
\frac{1}{\pi^{M}} \int e^{-H^{*}(I+A(j \omega+\beta)) H} d H=\frac{1}{\operatorname{det}(I+A(j \omega+\beta))}
$$

To see this, let $U \Lambda U^{*}$ denote the eigenvalue decomposition of the Hermitian matrix $A$. Now introduce the change of variables

$$
\tilde{H}=U^{*} H,
$$

then since $U$ is unitary

$$
d \tilde{H}=\left|\operatorname{det} U^{*}\right|^{2} d H=d H
$$

and the integral in (9) can be decomposed as

$$
\begin{array}{r}
\int d \tilde{H} e^{-\tilde{H}^{*}(I+\Lambda(j \omega+\beta)) \tilde{H}}= \\
\prod_{i=1}^{M} \int_{-\infty}^{\infty} e^{-\left(1+\beta \lambda_{i}+\lambda_{i} j \omega\right)|\tilde{H}(i)|^{2}} d \tilde{H}(i)
\end{array}
$$

For each $x$, we can choose $\beta$ such that $1+\beta \lambda_{i}>0$. With this choice of $\beta$, it is easy to see that [20]

$$
\frac{1}{\pi} \int_{-\infty}^{\infty} e^{-\left(1+\beta \lambda_{i}+\lambda_{i} j \omega\right)|\tilde{H}(i)|^{2}} d \tilde{H}(i)=\frac{1}{1+\lambda_{i}(j \omega+\beta)}
$$

We finally arrive at the result

$$
\frac{1}{\pi^{M}} \int d H e^{-H^{*}(I+A(j \omega+\beta)) H}=\prod_{i=1}^{M} \frac{1}{1+\lambda_{i}(y)(j \omega+\beta)}
$$

An alternative way to express the result above is

$$
\frac{1}{\pi^{M}} \int d H e^{-H^{*}(I+A(j \omega+\beta)) H}=\frac{1}{\operatorname{det}(I+A(j \omega+\beta))}
$$

which is the expression we arrived at in (9) intuitively by considering the left hand side as a formal Gaussian integral with correlation $(I+A(j \omega+\beta))^{-1}$.

\section{REFERENCES}

[1] G. G. Tziritas, "On the distribution of positive definite gaussain quadratic functions," IEEE Transaction on Information Theory, vol. IT-33, pp. 895-906, 1987.

[2] M. S. R. F. J. Reifler and K. Malakian, "Distribution of general noncentral positive definite quadratic form in k-dimensions," IEEE transactions on Aerosp. Electron. Syst., vol. 25, pp. 411-414, May 1989.

[3] A. M. Mathai and S. B. Provost, "Quadratic forms in random variables," New York: Marcel Dekker, 1992.

[4] J. Gurland, "Distribution of definite and indefinite quadratic forms," Ann. Math. Stat., vol. 26, pp. 122-127, 1955.

[5] H. E. Robbnis, "The distribution of a definite quadratic form," Ann. Math. Stat., vol. 19, pp. 266-270, 1948.

[6] B. K. Shah, "Distribution of definite and indefinite quadratic forms from a non-central normal distribution," Ann. Math. Stat., vol. 34, pp. 186$190,1963$.

[7] T. Hayakawa, "On the distribution of a quadratic form in a multivariate normal sample," Ann. Inst. Statist. Math., vol. 18, pp. 191-200, 1966.

[8] N. L. Johnson and S. Kotz, "Continuous univariate distributions," New York: Houghton Mifflin, vol. 2, 1970.

[9] J. P. Imhof, "Computing the distribution of quadratic forms in normal variates," Biometrika, vol. 48, pp. 419-426, 1961.
[10] S. J. Press, "Linear Combinations of noncentral chi-square variates," Ann. Math. Stat., vol. 37, pp. 480-487, 1966.

[11] A. Grad and H. Solomon, "Distribution of quadratic forms and some applications," Ann. Math. Stat., vol. 26, pp. 464-477, 1955.

[12] J. Robinson, "The distribution of a general quadratic form in normal variates," Australian J. Stat., vol. 7, pp. 110-114, 1965.

[13] Y. Y. Wang, "A comparision of several variance component estimators," Biometrika, vol. 54, pp. 301-305, 1967.

[14] K. H. Biyari and W. C. Lindsey, "Statistical distribution of hermitian quadratic forms in complex Gaussian variables," IEEE transactions on Inform. Theory, vol. 39, pp. 1076-1082, May 1993.

[15] J. Huang and L. L. Campbell, "Tail probability of a noncentral indefinite gaussian quadratic form," Comm. Stat. Simul., vol. 23, pp. 431-439, 1994.

[16] N. L. J. S. Kot and D. W. Boyd, "Series representation of distribution of quadratic forms in normal variables 1. central case," Ann. Math. Stat., vol. 38, pp. 832-837, 1967.

[17] D. Raphaeli, "Distribution of noncentral indefinite quadratic forms in complex normal variables," IEEE transactions on Information Theory, vol. 42, no. 3, pp. 1002-1007, May 1996.

[18] —_, "Noncoherent coded modulation," IEEE transactions on Communication, vol. 44, no. 2, pp. 172-183, Feb 1996.

[19] S. O. Rice, "Distribution of quadratic forms in normal variables: Evaluation by numerical integration," SIAM. J. Scient. Statist. Comput., vol. 1, pp. 438-448, Dec 1980

[20] Gradshteyn and Ryzhik, Table of Integrals, Series, and Products. Alan Jeffrey and Daniel Zwillinger (eds.), Seventh edition, Feb, 2007.

[21] T. Y. Al-Naffouri and A. H. Sayed, "Transient analysis of datanormalized adaptive filters, IEEE Trans. Signal Processing, vol. 51, No. 3, pp. 639-652, Mar. 2003

[22] T. Y. Al-Naffouri, M. Sharif, and B. Hassibi "How much does transmit correlation affect the sum-rate scaling of MIMO Gaussian broadcast channels?" IEEE Transactions on Communications, no. 2, Feb. 2009.

[23] T. Y. Al-Naffouri, M. Sharif, and B. Hassibi How much does transmit correlation affect the sum-rate if MIMO downlink channels? International Symposium on Information Theory, Seattle, OR, Jul. 2006.

[24] T. Y. Al-Naffouri and B. Hassibi, "On the Distribution of Indefinite Hermitian Quadratic Forms in Gaussian Random Variables," under preparation. 